\title{
VORTICES AND SPONTANEOUS SYMMETRY BREAKING IN ROTATING BOSE GASES
}

\author{
ROBERT SEIRINGER
}

\begin{abstract}
We present a rigorous proof of the appearance of quantized vortices in dilute trapped Bose gases with repulsive two-body interactions subject to rotation, which was obtained recently in joint work with Elliott Lieb [1]. Starting from the many-body Schrödinger equation, we show that the ground state of such gases is, in a suitable limit, well described by the nonlinear Gross-Pitaevskii equation. In the case of axially symmetric traps, our results show that the appearance of quantized vortices causes spontaneous symmetry breaking in the ground state.
\end{abstract}

\section{INTRODUCTION}

In recent remarkable experiments [2, 3, 4, 5], the appearance of quantized vortices in the ground state (and low temperature equilibrium states) of rotating dilute Bose gases was beautifully demonstrated. These quantized vortices are a consequence of the superfluid nature of the system under investigation. In particular, since the system is almost completely Bose condensed, it behaves like a single quantum particle.

The state of ultracold dilute Bose gases is usually described by means of the Gross-Pitaevskii (GP) equation [6, 7, 8, 9, 10, This non-linear Schrödinger equation originates as the variational equation from the corresponding GP energy functional, given by

$$
\mathcal{E}^{\mathrm{GP}}[\phi]=\left\langle\phi\left|H_{0}\right| \phi\right\rangle+4 \pi g \int_{\mathbb{R}^{3}}|\phi(x)|^{4} d^{3} x .
$$

Here, $\phi \in L^{2}\left(\mathbb{R}^{3}\right)$, and $H_{0}$ denotes the one-particle Hamiltonian, describing the kinetic, potential and rotational energy of the particles. In fact, if $\Omega$ denotes the angular velocity vector and $V(x)$ the trap potential, $H_{0}$ is, in appropriate units, given by

$$
H_{0}=-\Delta+V(x)-\Omega \cdot L
$$

where $L=-i x \wedge \nabla$ denotes the angular momentum operator. The parameter $g$ in (11) is nonnegative and measures the interaction strength among the particles. The trap potential $V(x)$ is assumed to be locally bounded and to

Plenary talk given at QMath10, $10^{\text {th }}$ Quantum Mathematics International Conference, Moeciu, Romania, September 10-15, 2007.

(c) 2008 by the author. This work may be reproduced, in its entirety, for noncommercial purposes. 
increase fast enough at infinity in order to have the particles confined to the trap (and, in particular, to ensure that $H_{0}$ is bounded from below). More precisely, we assume that

$$
\lim _{|x| \rightarrow \infty}\left(V(x)-\frac{1}{4}|\Omega \wedge x|^{2}\right)=+\infty .
$$

Since $-\Delta-\Omega \cdot L=(-i \nabla+\Omega \wedge x / 2)^{2}-|\Omega \wedge x|^{2} / 4$, this implies the desired property.

The GP energy is the minimal value of $\mathcal{E}^{\mathrm{GP}}[\phi]$ among all (appropriate normalized) functions $\phi$, i.e.,

$$
E^{\mathrm{GP}}(g, \Omega)=\inf _{\|\phi\|_{2}=1} \mathcal{E}^{\mathrm{GP}}[\phi] .
$$

Using (3) and the fact that $g \geq 0$, it is in fact not difficult to show that the infimum is actually a minimum (see [11]). That is, there exists a minimizer of the GP functional (11). Note that, in general, there may be many different minimizers. In any case, any minimizer satisfies the GP equation

$$
-\Delta \phi(x)+V(x) \phi(x)-\Omega \cdot L \phi(x)+8 \pi g|\phi(x)|^{2} \phi(x)=\mu \phi(x)
$$

where $\mu=E^{\mathrm{GP}}(g, \Omega)+4 \pi g \int_{\mathbb{R}^{3}}|\phi(x)|^{4} d^{3} x$ is the corresponding chemical potential.

For axially symmetric $V(x)$, i.e., in case $V(x)$ commutes with $\Omega \cdot L$, the GP functional is invariant under rotation about the $\Omega$ axis. It turns out that for any $\Omega \neq 0$, this rotational symmetry is broken in the GP minimizer for large enough interaction strength $g$ [12, 13]. This symmetry breaking is the result of the appearance of quantized vortices since, in case of more than one vortex, they cannot be arrange in a symmetric way. Note that, in particular, this implies that there will be many GP minimizers (for $g$ large enough).

We remark that the phenomenon just described is a special feature of rotating systems and cannot be observed in a non-rotating system. In fact, for $\Omega=0$ there is always a unique minimizer of the GP functional [1].

It turns out that the appearance of quantized vortices, and the resulting symmetry breaking, which we have just described, are not merely a property of the GP theory, but can actually be derived out of the underlying (manyparticle) Schrödinger equation. This was proved in [1]. In the following sections, we will give a summary of these results, and we will explain the key ideas leading to their proof.

\section{The Schrödinger Equation for Many Particles}

Consider a quantum-mechanical system of a large number, $N$, of bosons, with one-particle energies described by $H_{0}$ (given in (2) above). We assume that the particles interact via a repulsive pair interaction potential $v_{a}(x)$. 
The Hamiltonian for this system is given by

$$
H_{N}=\sum_{i=1}^{N} H_{0}^{(i)}+\sum_{1 \leq i<j \leq N} v_{a}\left(x_{i}-x_{j}\right),
$$

where the superscript $(i)$ refers to the fact that $H_{0}$ acts on the $i$ 'th variable. Since the particles under consideration are bosons, the Hamiltonian $H_{N}$ acts on the subspace of totally symmetric functions in $\bigotimes^{N} L^{2}\left(\mathbb{R}^{3}\right)$, which we denote by $\mathcal{H}_{N}$.

The interaction potential $v_{a}(x)$ is assumed to be nonnegative and of short range. More precisely, it is assumed to have finite scattering length [11, 14], denoted by $a$, which means that it has to be integrable at infinity (i.e., it has to decay faster than $|x|^{-3}$ ). A typical example would be a hard sphere interaction, which formally means that $v_{a}(x)=\infty$ for $|x| \leq a$ and $v_{a}(x)=0$ otherwise. We shall, in fact, choose some fixed (nonnegative) interaction potential $w(x)$ with scattering length 1 and obtain $v_{a}(x)$ by scaling as

$$
v_{a}(x)=a^{-2} w(x / a) .
$$

It is then easy to see that $v_{a}(x)$ has scattering length $a$. Moreover, $a$ now appears as a parameter in the Hamiltonian $H_{N}$, which can be freely varied. In particular, we can (and will) let $a$ depend on $N$. We note that this scaling of $v_{a}(x)$ is, of course, mathematically and physically equivalent to scaling the trap potential $V(x)$ (and the angular velocity $\Omega$ ) in an appropriate way, while keeping the interaction potential fixed.

2.1. Ground State Energy. For fixed $w(x)$ and $V(x)$, we shall denote the ground state energy of $H_{N}$ as $E_{0}(N, a, \Omega)$, i.e.,

$$
E_{0}(N, a, \Omega)=\inf _{\Psi \in \mathcal{H}_{N}} \frac{\left\langle\psi\left|H_{N}\right| \Psi\right\rangle}{\langle\Psi \mid \Psi\rangle} .
$$

Since the ground state energy per unit volume of a homogeneous Bose gas with interaction $v_{a}(x)$ at density $\rho$ is given by $4 \pi a \rho^{2}$ for low density [15], it is reasonable to expect that $E_{0}(N, a, \Omega) \approx N E^{\mathrm{GP}}(N a, \Omega)$ for dilute gases. Here, dilute means that $a^{3} \bar{\rho} \ll 1$, where $\bar{\rho}$ denotes the average density. This condition is, in particular, satisfied if $N \gg 1$ and $N a=O(1)$. We call this the GP limit. In this limit, we have the following result [1].

THEOREM 1. For any $g \geq 0$ and $\Omega \in \mathbb{R}^{3}$,

$$
\lim _{N \rightarrow \infty} \frac{E_{0}(N, g / N, \Omega)}{N}=E^{\mathrm{GP}}(g, \Omega)
$$

That is, for large $N$ and $a=O(1 / N)$, the ground state energy per particle is given by the GP energy with coupling parameter $g=N a$. Theorem 1 holds for all angular velocities $\Omega$ (satisfying the stability criterion (3)). It extends previous results in the nonrotating case $\Omega=0$ [11.

Note that the right side of (5) is independent of the choice of the unscaled interaction potential $w(x)$. In the dilute limit considered here, only the 
scattering length $a$ matters, and not the details of the interaction potential. Note also that the result cannot be obtained by simple perturbation theory; in fact, the $\int|\phi|^{4}$ term in the GP functional is partly kinetic energy, and not the average value of $v_{a}(x)$ (which might even be zero, as in the case of the hard-sphere interaction).

As will be pointed out in Subsect. 2.3 below, it is essential to restrict to symmetric wave functions (bosons) in Theorem 1. For the absolute ground state energy (defined as the infimum of $H_{N}$ over all wavefunctions, not necessarily symmetric ones), the result is wrong, in general. For the absolute ground state, the right side has to be replaced by minimizing a densitymatrix functional instead [13].

2.2. Bose-Einstein Condensation. The GP energy functional (11) and its minimizers contain information not only about the ground state energy of the many-body Hamiltonian (44), but also about the ground state or, more precisely, its reduced density matrices. Recall that for any wavefunction $\Psi \in \mathcal{H}_{N}$, its reduced one-particle density matrix $\gamma_{N}^{(1)}$ is given by the kernel

$$
\gamma_{N}^{(1)}\left(x, x^{\prime}\right)=N \int_{\mathbb{R}^{3(N-1)}} \Psi\left(x, x_{2}, \ldots, x_{N}\right) \Psi^{*}\left(x^{\prime}, x_{2}, \ldots, x_{N}\right) d^{3} x_{2} \cdots d^{3} x_{N} .
$$

Note that this defines a positive trace class operator on the one-particle space $L^{2}\left(\mathbb{R}^{3}\right)$.

The one-particle density matrix of a state $\Psi$ contains all the information about the system concerning expectation values of one-particle operators. It particular, the concept of Bose-Einstein condensation (BEC) is defined in terms of $\gamma_{N}^{(1)}$.

Note that if $\Psi$ is normalized, i.e., $\|\Psi\|_{2}=1$, then the trace of $\gamma_{N}^{(1)}$ is $N$. BEC means that $\gamma_{N}^{(1)}$ has an eigenvalue of order $N$. The corresponding eigenfunction is called the condensate wave function. For dilute systems, as we consider here, one expects in fact complete $B E C$, meaning that $\gamma_{N}^{(1)}$ is approximately a rank one projection, or $\gamma_{N}^{(1)}\left(x, x^{\prime}\right) \approx N \phi(x) \phi\left(x^{\prime}\right)$ for some normalized $\phi \in L^{2}\left(\mathbb{R}^{3}\right)$.

In the non-rotating case $\Omega=0$, complete BEC in the ground state of $H_{N}$ was proved in [16]. Moreover, it was shown that the condensate wave function equals the GP minimizer. Recall that in the case $\Omega=0$ there is a unique minimizer of the GP functional (1) (up to constant phase factor, of course), which we denote by $\phi^{\mathrm{GP}}$. That is, if $\gamma_{N}^{(1)}$ denotes the one-particle density matrix of the ground state $\Psi$ of $H_{N}$ for $\Omega=0$, then

$$
\lim _{N \rightarrow \infty} \frac{1}{N} \gamma_{N}^{(1)}\left(x, x^{\prime}\right)=\phi^{\mathrm{GP}}(x) \phi^{\mathrm{GP}}\left(x^{\prime}\right)
$$

in the GP limit $N \rightarrow \infty, N a \rightarrow g$. To be precise, the limit (6) holds in trace norm sense. Note that although $a$ is scaled to zero in the limit considered, the right side of (6) depends on $g=N a$ via $\phi^{\mathrm{GP}}$. 
The corresponding result for $\Omega \neq 0$ is necessarily more complicated because of non-uniqueness of the GP minimizer $\phi^{\mathrm{GP}}$. It is actually more natural to not just look at a ground state of $H_{N}$ (which may not be unique in the rotating case either), but on the set of all approximate ground states. These are defined as sequences of (bosonic) $N$-particle density matrices $\gamma_{N}$ (that is, positive operators on $\mathcal{H}_{N}$ with trace one) with $\operatorname{Tr} H_{N} \gamma_{N} \approx N E^{\mathrm{GP}}$. One can then expect that the reduced one-particle density matrix $\gamma_{N}^{(1)}$ of any such approximate ground state is a convex combination of GP minimizers, i.e.,

$$
\gamma_{N}^{(1)}\left(x, x^{\prime}\right) \approx \sum_{i} \lambda_{i} \phi_{i}^{\mathrm{GP}}(x) \phi_{i}^{\mathrm{GP}}\left(x^{\prime}\right)^{*}
$$

where each $\phi_{i}^{\mathrm{GP}}$ is a GP minimizer, and $\sum_{i} \lambda_{i}=N$.

Theorem 2 below states that this is indeed the case. The mathematically precise formulation is slightly complicated by the fact that the set of GP minimizers is, in general, not countable.

Let $\Gamma$ be the set of all limit points of one-particle density matrices of approximate ground states:

$$
\begin{gathered}
\Gamma=\left\{\gamma: \exists \text { sequence } \gamma_{N}, \lim _{N \rightarrow \infty, N a \rightarrow g} \frac{1}{N} \operatorname{Tr} H_{N} \gamma_{N}=E^{\mathrm{GP}}(g, \Omega),\right. \\
\left.\lim _{N \rightarrow \infty} \frac{1}{N} \gamma_{N}^{(1)}=\gamma\right\} .
\end{gathered}
$$

Since $H_{0}$ has a compact resolvent by our assumption (3), one easily sees that $\operatorname{Tr} \gamma=1$ for all $\gamma \in \Gamma$. Moreover, because of the linearity of the conditions in (7), $\Gamma$ is clearly convex.

THEOREM 2. For given value of $g \geq 0$ and $\Omega$, let $\Gamma$ denote the set of all limit points of one-particle density matrices of approximate ground states of $H_{N}$, defined in (7).

(i) $\Gamma$ is a compact and convex subset of the set of all trace class operators.

(ii) Let $\Gamma_{\text {ext }} \subset \Gamma$ denote the set of extreme points in $\Gamma$. We have $\Gamma_{\text {ext }}=$ $\left\{|\phi\rangle\langle\phi|: \mathcal{E}^{\mathrm{GP}}[\phi]=E^{\mathrm{GP}}(g, \Omega)\right\}$, i.e., the extreme points in $\Gamma$ are given by the rank-one projections onto GP minimizers.

(iii) For each $\gamma \in \Gamma$, there is a positive (regular Borel) measure $d \mu_{\gamma}$, supported in $\Gamma_{\text {ext }}$, with $\int_{\Gamma_{\mathrm{ext}}} d \mu_{\gamma}(\phi)=1$, such that

$$
\gamma=\int_{\Gamma_{\text {ext }}} d \mu_{\gamma}(\phi)|\phi\rangle\langle\phi|
$$

where the integral is understood in the weak sense. That is, every $\gamma \in \Gamma$ is a convex combination of rank-one projections onto GP minimizers.

We remark that item (iii) of Theorem 2 follows from item (ii) by Choquet's Theorem [17]. 
As explained above, Theorem 2 is the natural analogue of (즈) in the rotating case. It can also be interpreted as a rigorous proof of superfluidity. As typical for superfluids, angular momentum in rotating systems is acquired in terms of quantized vortices. These can be seen by solving the GP equation.

Theorem 2 also shows the occurrence of spontaneous symmetry breaking. As remarked earlier, axial symmetry of the trap $V(x)$ leads to nonuniqueness of the GP minimizer for $g$ large enough [12, 13. Uniqueness can be restored by perturbing $H_{0}$ to break the symmetry and favor one of the minimizers. This then leads to complete BEC in the usual sense, since $\Gamma$ contains contains only one element in case the GP functional (1) has a unique minimizer.

As in the case of the ground state energy discussed in the previous subsection, the situation is very different for the absolute ground state. The set $\Gamma$ consists of only one element in this case (namely the minimizer of the density matrix functional discussed below, which is unique for any value of $\Omega$ and $g$ ). In particular, there is no spontaneous symmetry breaking in the absolute ground state. This will be discussed in the next subsection.

2.3. The Absolute Ground State. Let $E_{\text {abs }}(N, a, \Omega)$ denote the absolute ground state energy of $H_{N}$ in (4), irrespective of symmetry constraints, i.e.,

$$
E_{\text {abs }}(N, a, \Omega)=\inf _{\Psi \in L^{2}\left(\mathbb{R}^{3 N}\right)} \frac{\left\langle\psi\left|H_{N}\right| \Psi\right\rangle}{\langle\Psi \mid \Psi\rangle} .
$$

Note that necessarily $E_{\text {abs }}(N, a, \Omega) \leq E_{0}(N, a, \Omega)$. As is well known, for $\Omega=0$ the two energies are equal. This turns out not to be the case for $\Omega \neq 0$, in general.

In the GP limit, the absolute ground state energy, and the corresponding one-particle reduced density matrices of approximate ground states, turn out to be described by a GP density matrix functional, introduced in [12],

$$
\mathcal{E}^{\mathrm{DM}}[\gamma]=\operatorname{Tr}\left[H_{0} \gamma\right]+4 \pi g \int_{\mathbb{R}^{3}} \rho_{\gamma}(x)^{2} d^{3} x .
$$

Here, $\gamma$ is a positive trace class operator on $L^{2}\left(\mathbb{R}^{3}\right)$, and $\rho_{\gamma}$ denotes the density of $\gamma$, i.e., $\rho_{\gamma}(x)=\gamma(x, x)$. The functional $\mathcal{E}^{\mathrm{DM}}$ can be shown 12 to have a unique minimizer (under the normalization condition $\operatorname{Tr} \gamma=1$ ), which we denote by $\gamma^{\mathrm{DM}}$. We denote the corresponding energy by $E^{\mathrm{DM}}(g, \Omega)=$ $\mathcal{E}^{\mathrm{DM}}\left[\gamma^{\mathrm{DM}}\right]$.

The following Theorem concerning the absolute ground state of $H_{N}$ was proved in [13.

THEOREM 3. For any fixed $g \geq 0$ and $\Omega \in \mathbb{R}^{3}$,

$$
\lim _{N \rightarrow \infty} \frac{E_{\mathrm{abs}}(N, g / N, \Omega)}{N}=E^{\mathrm{DM}}(g, \Omega) \quad \text { and } \quad \lim _{N \rightarrow \infty} \frac{1}{N} \gamma_{\text {abs }}^{(1)}=\gamma^{\mathrm{DM}}
$$

Here, $\gamma_{\text {abs }}^{(1)}$ denotes the one-particle density matrix of any approximate (absolute) ground state sequence of $H_{N}$. In other words, the set $\Gamma$ defined 
as in (7), but for the absolute ground state, contains only one element, namely the unique minimizer of $\mathcal{E}^{\mathrm{DM}}$.

Note that $\mathcal{E}^{\mathrm{GP}}$ is the restriction of $\mathcal{E}^{\mathrm{DM}}$ to rank one projections. In the case of symmetry breaking (i.e., for $g$ large enough), rank $\gamma^{\mathrm{DM}} \geq 2$, and hence $E^{\mathrm{DM}}<E^{\mathrm{GP}}$. In particular, in view of Theorems 1 3 , the absolute and bosonic ground state differ significantly, in general, both in terms of their energy and their reduced one-particle density matrix.

We remark that the results explained in this subsection become physically relevant if one considers bosons with internal degrees of freedom. Internal degrees of freedom effectively increase the number of allowed symmetry classes (see, e.g., 18). In particular, if the number of states of the internal degrees of freedom of the bosons is greater or equal to the rank of $\gamma^{\mathrm{DM}}$, $E^{\mathrm{DM}}(g, \Omega)$ equals the (bosonic) ground state energy per particle in the GP limit. More generally, one can show that in the GP limit the functional $\mathcal{E}^{\mathrm{DM}}$, when restricted to density matrices of rank at most $n$, correctly describes the ground state energy (and corresponding one-particle density matrix) of bosons with $n$ internal states.

\section{Sketch of the Proof of Theorem 1}

In the following, we shall give a brief outline of the main ideas in the proof of Theorem 1, For details we refer to the original work in [1]. We shall restrict our attention to the appropriate lower bound on the ground state energy $E_{0}(N, a, \Omega)$. The corresponding upper bound can be obtained via a variational argument, as explained in [13.

A convenient way to keep track of the bosonic symmetry requirement is to work in Fock space. Recall that the bosonic Fock space $\mathcal{F}$ is given by $\mathcal{F}=\bigoplus_{N \geq 0} \mathcal{H}_{N}$. In terms of creation and annihilation operators $a_{j}^{\dagger}$ and $a_{j}$ on $\mathcal{F}$, the Hamiltonian can be written as

$$
H=\sum_{j \geq 1} e_{j} a_{j}^{\dagger} a_{j}+\frac{1}{2} \sum_{i j k l} a_{i}^{\dagger} a_{j}^{\dagger} a_{k} a_{l} W_{i j k l} .
$$

Here, we choose the basis in the one-particle space $L^{2}\left(\mathbb{R}^{3}\right)$ as to diagonalize $H_{0}$, i.e., $H_{0}=\sum_{j} e_{j}\left|\varphi_{j}\right\rangle\left\langle\varphi_{j}\right|$, and $a_{j}^{\dagger}$ creates a particle with wavefunction $\varphi_{j}$, whereas $a_{j}$ annihilates it. The coefficients $W_{i j k l}$ are given in terms of expectation values of $v_{a}(x)$, namely $W_{i j k l}=\left\langle\varphi_{i} \otimes \varphi_{j}\left|v_{a}\right| \varphi_{k} \otimes \varphi_{l}\right\rangle$.

Note that $H$ in (18) commutes with total particle number operator $\sum_{j \geq 1} a_{j}^{\dagger} a_{j}$. Hence it splits into a direct sum of operators on $\mathcal{H}_{N}$ for $N=0,1, \ldots$. In fact, our $H_{N}$ in (4) is just the restriction of $H$ to $\mathcal{H}_{N}$.

The analysis employed for obtaining a lower bound on the ground state energy of $H$ in the sector of $N$ particles consists of two main steps:

1. Eq. (8) is not necessarily well defined. E.g., if $v_{a}(x)$ is the hard-core interaction potential (or, more generally, is not integrable), then $W_{i j k l}=\infty$ for any set of indices. In order to overcome this problem, we shall first show that, for a lower bound, one can replace $v_{a}(x)$ by 
a "soft" and longer ranged potential $U(x)$ (with the same scattering length), at the expense of the high-momentum part of the kinetic energy. We note that this step is necessary even in the case when $v_{a}(x)$ is integrable (and hence (8) is well defined) in order to proceed with the second step.

2. After having replaced $v_{a}(x)$ by the softer potential $U(x)$, one then shows that it is possible to replace the operators $a_{j}^{\dagger}$ and $a_{j}$ by complex numbers $z_{j}$ without changing the ground state energy too much [19]. Note that if all the $a_{j}^{\dagger}$ and $a_{j}$ in (8) are treated as numbers, the expression (8) looks very similar to the GP energy functional (11); in fact, it is given by

$$
\left\langle\phi_{\mathbf{z}}\left|H_{0}\right| \phi_{\mathbf{z}}\right\rangle+\frac{1}{2} \int_{\mathbb{R}^{6}} v_{a}(x-y)\left|\phi_{\mathbf{z}}(x)\right|^{2}\left|\phi_{\mathbf{z}}(y)\right|^{2} d^{3} x d^{3} y,
$$

with $\phi_{\mathbf{z}}(x)=\sum_{j} z_{j} \varphi_{j}(x)$.

In the following, we shall explain these two main steps in more detail.

3.1. Step 1: Generalized Dyson Lemma. The following Lemma can be viewed as a generalization of an idea of Dyson [20]. The purpose of the lemma is give a lower bound on the interaction potential $v_{a}(x)$ in terms of a softer and longer ranged potential $U(x)$, at the expense of some kinetic energy (see also [15]). For our purpose, we can only spare the high momentum part of the kinetic energy, however; the low momentum part is needed for the $H_{0}$ term in the GP functional.

We thus have to separate the high momentum from the low momentum part of the kinetic energy. This can be done in the following way. The proof of Lemma 1 is given in [21].

Lemma 1. Let $v_{a}(x)$ have scattering length $a$ and range $R_{0}$. Let $\theta_{R}$ be the characteristic function of the ball $\{x:|x|<R\}$. Let $0 \leq \chi(p) \leq 1$, such that $h(x) \equiv \widehat{1-\chi}(x)$ is bounded and integrable,

$$
f_{R}(x)=\sup _{|y| \leq R}|h(x-y)-h(x)|,
$$

and

$$
w_{R}(x)=\frac{2}{\pi^{2}} f_{R}(x) \int_{\mathbb{R}^{3}} f_{R}(y) d^{3} y .
$$

Then for any $\varepsilon>0$ and any positive radial function $U(x)$ supported in $R_{0} \leq|x| \leq R$ with $\int U=4 \pi$ we have the operator inequality

$$
-\nabla \chi(p) \theta_{R}(x) \chi(p) \nabla+\frac{1}{2} v_{a}(x) \geq(1-\varepsilon) a U(x)-\frac{a}{\varepsilon} w_{R}(x) .
$$

Here, $\chi(p)$ denotes a multiplication operator in momentum space. Note that the operator $-\nabla \chi(p) \theta_{R}(x) \chi(p) \nabla$ can be interpreted as a Laplacian that has been localized to the ball of radius $R$ and cut off in momentum space. Because of the cut-off, this is not a local operator, however. The parameter $R$ is chosen such that $a \ll R \ll N^{-1 / 3}$. Note that to leading 
order in $a / R$, the scattering length of $2 a U(x)$ is given in terms of its first order Born approximation as $(8 \pi)^{-1} a \int_{\mathbb{R}^{3}} U(x) d^{3} x=a$.

Because of the appearance of the characteristic function $\theta_{R}(x)$ in (9), Lemma 1 has the following immediate consequence. If $y_{1}, \ldots, y_{n}$ are $n$ points in $\mathbb{R}^{3}$ whose mutual distance is at least $2 R$, then

$$
-\nabla \chi(p)^{2} \nabla+\frac{1}{2} \sum_{i=1}^{n} v_{a}\left(x-y_{i}\right) \geq \sum_{i=1}^{n}\left[(1-\varepsilon) a U\left(x-y_{i}\right)-\frac{a}{\varepsilon} w_{R}\left(x-y_{i}\right)\right] .
$$

This bound accomplishes the replacement of the hard interaction potential $v_{a}(x)$ by a soft one, at the expense of the high momentum part of the kinetic energy. For given configuration of $N-1$ particles, this estimate is applied to the remaining particle. Of course one still has to estimate the contribution from configurations where 2 (or more) of the $N-1$ fixed particles are closer together than $2 R$. This can be achieved by a FeynmanKac integral representation [22] of the ground state. We refer to [1] for details.

3.2. Step 2: Coherent States. The Fock space $\mathcal{F}$ can be viewed as an infinite tensor product of the form $\mathcal{F}=\bigotimes_{j \geq 1} \mathcal{F}_{j}$, with $\mathcal{F}_{j}$ spanned by the vectors $\left(a_{j}^{\dagger}\right)^{n}|0\rangle$ for $n=0,1, \ldots$ Here, $|0\rangle$ denotes the Fock space vacuum.

Consider first the case of a single mode, $\mathcal{F}_{1}$, say. For $z \in \mathbb{C}$, a coherent state [23] in $\mathcal{F}_{1}$ is defined by

$$
|z\rangle=e^{-|z|^{2} / 2+z a_{1}^{\dagger}}|0\rangle \text {. }
$$

These states span in the whole space $\mathcal{F}_{1}$. In fact, they satisfy the completeness relation

$$
\int_{\mathbb{C}} d z|z\rangle\langle z|=\mathbb{I}
$$

where $d z$ stands for $\pi^{-1} d x d y$, and $z=x+i y, x, y \in \mathbb{R}$.

In terms of coherent states, upper and lower symbols of operators can be defined. Lower symbols are simply the expectation values of operators in coherent states, e.g., $\left\langle z\left|a_{1}\right| z\right\rangle=z$ and $\left\langle z\left|a_{1}^{\dagger} a_{1}\right| z\right\rangle=|z|^{2}$. Upper symbols, on the other hand, represent functions of $z$ which, when integrated against $|z\rangle\langle z| d z$ over $\mathbb{C}$, yield given operators. For instance, it is not difficult to see that $a_{1}=\int d z z|z\rangle\langle z|$, while $a_{1}^{\dagger} a_{1}=\int d z\left(|z|^{2}-1\right)|z\rangle\langle z|$. Hence, upper and lower symbols of $a_{1}$ are given by $z$, whereas the lower symbol of $a_{1}^{\dagger} a_{1}$ is $|z|^{2}$ and the upper symbol is $|z|^{2}-1$.

Note that lower symbols yield upper bounds on ground state energies, by the variational principle, while upper symbols are useful for lower bounds. The difference in the symbols thus quantifies the error one makes in replacing the operators $a_{1}^{\dagger}$ and $a_{1}$ by numbers. In particular, for every quadratic term $a_{1}^{\dagger} a_{1}$ a factor -1 has to be taken into account. For this reason, one cannot introduce coherent states of all the modes $j$, but only for a finite number of them. 
In fact, we shall introduce coherent states of all the modes $1 \leq j \leq J$ for some $J \gg 1$. That is, we first write $\mathcal{F}=\mathcal{F}_{<} \otimes \mathcal{F}_{>}$, where $\mathcal{F}_{<}$is spanned by the vectors of the form $\left(a_{1}^{\dagger}\right)^{n_{1}} \cdots\left(a_{J}^{\dagger}\right)^{n_{J}}|0\rangle$, with $n_{j} \in \mathbb{N}$ for $1 \leq j \leq J$. For $\mathbf{z}=\left(z_{1}, \ldots, z_{J}\right) \in \mathbb{C}^{J}$, we introduce the projection operator $\Pi(\mathbf{z})$ on $\mathcal{F}_{<}$, given by

$$
\Pi(\mathbf{z})=\left|z_{1} \otimes \cdots \otimes z_{J}\right\rangle\left\langle z_{1} \otimes \cdots \otimes z_{J}\right| .
$$

Using upper symbols, we can then write the Hamiltonian $H$ in (8) as

$$
H=\int_{\mathbb{C}^{J}} d \mathbf{z} \Pi(\mathbf{z}) \otimes h(\mathbf{z}) .
$$

Here, $h(\mathbf{z})$ represents the upper symbol of $H$. Since only the modes $1 \leq$ $j \leq J$ have been replaced by numbers, $h(\mathbf{z})$ is an operator on $\mathcal{F}_{>}$. Using the completeness property of the coherent states, Eq. (10), it is then easy to see that

$$
\inf \operatorname{spec} H \geq \inf _{\mathbf{z}} \inf \operatorname{spec} h(\mathbf{z}) .
$$

One then proceeds to show that $h(\mathbf{z}) \approx \mathcal{E}^{\mathrm{GP}}\left[\phi_{\mathbf{z}}\right]$ modulo controllable error terms. These error terms are, in fact, operators on $\mathcal{F}_{>}$which describe both the interactions among particles in high modes as well as the interaction between particles in modes $j \leq J$ and $j>J$. Precise bounds on these terms can be found in [1].

\section{Sketch of the Proof of Theorem 2}

In order to obtain information on (approximate) ground states from bounds on the energy, one proceeds as follows. One first perturbs the Hamiltonian $H_{N}$ in (44) by some one-particle perturbation $S$, and applies the same perturbation to the GP functional (1). One then shows that the result of Theorem 1 still holds for the perturbed system. In fact, the proof of Theorem 1 outlined in the previous section is sufficiently robust in order to easily incorporate such a modification.

Griffiths' argument 24] then implies that, for any $\gamma \in \Gamma$, and any bounded hermitian operator $S$,

$$
\operatorname{Tr} S \gamma \geq \min _{\phi=\phi^{\mathrm{GP}}}\langle\phi|S| \phi\rangle,
$$

where the minimum on the right side is taken over all GP minimizers. Inequality (11) is the key to the proof of Theorem 2. The rest follows from convexity theory [25], as we shall explain now.

Recall that an exposed point of a convex set $\mathcal{C}$ is an extreme point $p$ with the additional property that there is a tangent plane to $\mathcal{C}$ containing $p$ but no other point of $\mathcal{C}$. Hence, for $\widetilde{\gamma} \in \Gamma$ an exposed point, there exists an $S$ such that

$$
\operatorname{Tr} S \widetilde{\gamma} \leq \operatorname{Tr} S \gamma \quad \text { for all } \gamma \in \Gamma .
$$

with equality if and only if $\gamma=\widetilde{\gamma}$.

It is not very difficult to show that $\left|\phi^{\mathrm{GP}}\right\rangle\left\langle\phi^{\mathrm{GP}}\right| \in \Gamma$ for any GP minimizer $\phi^{\mathrm{GP}}$. Hence, if we choose $\gamma$ in (12) to be equal to $\left|\phi^{\mathrm{GP}}\right\rangle\left\langle\phi^{\mathrm{GP}}\right|$ for the $\phi^{\mathrm{GP}}$ 
that minimizes the right side of (11) for this particular $S$, the inequalities (11) and (12) imply that

$$
\min _{\phi=\phi^{\mathrm{GP}}}\langle\phi|S| \phi\rangle=\left\langle\phi^{\mathrm{GP}}|S| \phi^{\mathrm{GP}}\right\rangle \leq \operatorname{Tr} S \widetilde{\gamma} \leq \operatorname{Tr} S \gamma=\left\langle\phi^{\mathrm{GP}}|S| \phi^{\mathrm{GP}}\right\rangle
$$

and hence there is actually equality in (12). This, in turn, implies that $\widetilde{\gamma}=\left|\phi^{\mathrm{GP}}\right\rangle\left\langle\phi^{\mathrm{GP}}\right|$. We have thus shown that all exposed points of $\Gamma$ are of this form!

In order to extend this result to all extreme points, now merely exposed points, we employ Straszewicz's Theorem [25], which states that the exposed points are a dense subset of the extreme points. Strictly speaking, this theorem only holds in finite dimensions and not, a priori, in the infinite dimensional case under consideration here. However, because of compactness, the set $\Gamma$ is "almost" finite dimensional, and hence the theorem can be applied via an approximation argument. We refer again to [1] for details.

\section{Conclusions}

We have presented a rigorous justification of the Gross-Pitaevskii approximation for sufficiently dilute rotating Bose gases. For large particle number $N$ and both $N a$ and $\Omega$ of order 1 , the ground state of a rotating Bose gas is well approximated by the solution to the GP equation. This is true both for the energy and the reduced density matrices. In particular, our analysis proves the appearance of quantized vortices and the occurrence of spontaneous symmetry breaking in the parameter regime where these phenomena can be observed in the GP equation, e.g., for $\Omega \neq 0$ and $g$ large enough.

We point out that one of the major open problems in this field is the validity of the GP equation for rapidly rotating gases, where either $|\Omega| \rightarrow \infty$ as $N \rightarrow \infty$ (in case the trap potential grows faster than quadratic at infinity), or $\Omega$ approaches the trap frequency (for traps that are asymptotically quadratic). There is evidence that the GP descriptions breaks down once the number of vortices in the system is of the same order as the number of particles. Despite recent progress in this direction [26], a proof of this assertion is still lacking.

\section{ACKNOWLEDGMENTS}

Partial support by U.S. National Science grant PHY 0652356 and by an A.P. Sloan Fellowship is gratefully acknowledged.

\section{REFERENCES}

[1] E.H. Lieb and R. Seiringer, Derivation of the Gross-Pitaevskii Equation for Rotating Bose Gases, Commun. Math. Phys. 264, 505-537 (2006).

[2] J.R. Abo-Shaeer, C. Raman, J. M. Vogels, and W. Ketterle, Observation of Vortex Lattices in Bose-Einstein Condensates, Science 292, 476-479 (2001).

[3] E. Hodby, G. Hechenblaikner, S.A. Hopkins, O.M. Maragò, and C.J. Foot, Vortex Nucleation in Bose-Einstein Condensates in an Oblate, Purely Magnetic Potential, Phys. Rev. Lett. 88, 010405 (2001). 
[4] K.W. Madison, F. Chevy, W. Wohlleben, and J. Dalibard, Vortex Formation in a Stirred Bose-Einstein Condensate, Phys. Rev. Lett. 84, 806 (2000).

[5] M.R. Matthews, B.P. Anderson, P.C. Haljan, D.S. Hall, C.E. Wieman, and E.A. Cornell, Vortices in a Bose-Einstein Condensate, Phys. Rev. Lett. 83, 2498 (1999).

[6] D.A. Butts and D.S. Rokhsar, Predicted signatures of rotating Bose-Einstein condensates, Nature 397, 327-329 (1999).

[7] Y. Castin and R. Dum, Bose-Einstein condensates with vortices in rotating traps, Eur. Phys. J. D 7, 399-412 (1999).

[8] J.J. García-Ripoll and V.M. Pérez-García, Stability of vortices in inhomogeneous Bose condensates subject to rotation: A three-dimensional analysis, Phys. Rev. A 60, 48644874 (1999).

[9] A.L. Fetter and A.A. Svidzinsky, Vortices in a trapped dilute Bose-Einstein condensate, J. Phys.: Condens. Matter 13, R135-R194 (2001).

[10] A. Aftalion, Vortices in Bose-Einstein condensates, Progress in nonlinear differential equations and their applications, Vol. 67, Birkhäuser (2006).

[11] E.H. Lieb, R. Seiringer, and J. Yngvason, Bosons in a Trap: A Rigorous Derivation of the Gross-Pitaevskii Energy Functional, Phys. Rev. A 61, 043602 (2000).

[12] R. Seiringer, Gross-Pitaevskii Theory of the Rotating Bose Gas, Commun. Math. Phys. 229, 491-509 (2002).

[13] R. Seiringer, Ground state asymptotics of a dilute, rotating gas, J. Phys. A: Math. Gen. 36, 9755-9778 (2003).

[14] E.H. Lieb, R. Seiringer, J.P. Solovej, and J. Yngvason, The Mathematics of the Bose Gas and its Condensation, Oberwolfach Seminars, Vol. 34, Birkhäuser (2005).

[15] E.H. Lieb and J. Yngvason, Ground State Energy of the Low Density Bose Gas, Phys. Rev. Lett. 80, 2504-2507 (1998).

[16] E.H. Lieb and R. Seiringer, Proof of Bose-Einstein Condensation for Dilute Trapped Gases, Phys. Rev. Lett. 88, 170409 (2002).

[17] G. Choquet, Lectures on Analysis, vols. 1 and 2, W.A. Benjamin (1969).

[18] E. Eisenberg and E.H. Lieb, Polarization of interacting bosons with spin, Phys. Rev. Lett. 89, 220403 (2002).

[19] E.H. Lieb, R. Seiringer, and J. Yngvason, Justification of c-Number Substitutions in Bosonic Hamiltonians, Phys. Rev. Lett. 94, 080401 (2005).

[20] F.J. Dyson, Ground State Energy of a Hard-Sphere Gas, Phys. Rev. 106, 20-26 (1957).

[21] E.H. Lieb, R. Seiringer, and J.P. Solovej, Ground-state energy of the low-density Fermi gas, Phys. Rev. A 71, 053605-1-13 (2005).

[22] B. Simon, Functional Integration and Quantum Physics, Academic Press (1979).

[23] J. Klauder and B.-S. Skagerstam, Coherent states, applications in physics and mathematical physics, World Scientific (1985).

[24] R.B. Griffiths, A Proof that the Free Energy of a Spin System is Extensive, J. Math. Phys. 5, 1215-1222 (1964).

[25] R.T. Rockafellar, Convex Analysis, Princeton University Press (1970).

[26] J.-B. Bru, M. Correggi, P. Pickl, and J. Yngvason, The TF Limit for Rapidly Rotating Bose Gases in Anharmonic Traps, Preprint arXiv:0705.1107] [math-ph], Commun. Math. Phys. (in press).

Department of Physics, Princeton University, Jadwin Hall, Princeton NJ 08542-0708, USA

E-mail address: rseiring@princeton.edu 